\title{
HDAC and Ku70 axis- an effective target for apoptosis induction by a new 2-cyano-3- oxo-1,9-dien glycyrrhetinic acid analogue
}

Ping Gong ${ }^{1}$, Kun $\mathrm{Li}^{2}$, Ying $\mathrm{Li}^{1}$, Dan $\mathrm{Liu}^{2,3}$, Linxiang Zhao ${ }^{2,3}$ and Yongkui Jing ${ }^{1,3}$

\begin{abstract}
Methyl 2-cyano-3,12-dioxo-18ß-olean-1,9(11)-dien-30-oate (CDODO-Me, 10d) derived from glycyrrhetinic acid and methyl-2-cyano-3,12-dioxooleana-1,9-dien-28-oic acid (CDDO-Me) derived from oleanoic acid are potent apoptosis inducers developed to clinical trials. Both compounds have high affinity for reduced glutathione (GSH), which needs to be overcome to improve their target selectivity. We generated a new 10d analogue methyl 2-cyano-3-oxo-18 $\beta$ olean-1,9(11), 12-trien-30-oate (COOTO, 10e), which retains high apoptosis inducing ability, while displaying decreased affinity for GSH, and explored the acting targets. We found that it induces Noxa level, reduces c-Flip level and causes Bax/Bak activation. Silencing of either Noxa or Bak significantly attenuated apoptosis induction of 10e. We linked these events due to targeting HDAC3/HDAC6 and Ku70 axis. 10e treatment reduced the levels of HDAC3 and HDAC6 with increased DNA damage/repair marker gamma-H2AX ( $(-\mathrm{H} 2 \mathrm{AX})$ and acetylated Ku70. c-Flip dissociates from acetylated Ku70 undergoing degradation, while Bax dissociates from acetylated Ku70 undergoing activation. Silencing of either HDAC3 or HDAC6 enhanced 10e-induced apoptosis. We reveal a new action cascade of this category of compounds that involves targeting of HADC3/6 proteins and Ku70 acetylation.
\end{abstract}

\section{Introduction}

$18 \beta$-glycyrrhetinic acid (GA) is a naturally occurring oleanane-type pentacyclic triterpenoid isolated from the plant (Glycyrrhiza glabra) with antitumor activities ${ }^{1}$. We and other groups have performed structural modifications of this compound and obtained a derivative (methyl-2cyano-3,12-dioxo-18ß-olean-1,9(11)-dien-30-oate) (CDODO$\mathrm{Me})$ (Fig. 1a), with greater potency of apoptosis induction and tumor cell growth inhibition ${ }^{2-5}$. A similar compound, methyl2-cyano-3,12-dioxooleana-1,9-dien-28-oic acid (CDDO-Me) was also derived from oleanoic acid and is currently being clinically tested as an anticancer agent ${ }^{6,7}$. The mechanism by which both CDODO-Me and CDDO-Me inhibit tumor

\footnotetext{
Correspondence: Yongkui Jing (jingyongkui@syphu.edu.cn)

${ }^{1}$ Department of Pharmacology, Shenyang Pharmaceutical University,

Shenyang 110016, PR China

${ }^{2}$ Department of Medicinal Chemistry, Shenyang Pharmaceutical University,

Shenyang 110016, PR China

Full list of author information is available at the end of the article.

Edited by R.A. Knight
}

growth and induce apoptosis ${ }^{4,6,8}$ has not been elucidated. Both compounds bind strongly to reduced glutathione (GSH) and deplete intracellular $\mathrm{GSH}^{4,9-12}$, which has been originally assumed to be one mechanism of apoptosis induction. GSH protects the cells from oxidative stress ${ }^{13}$. High concentrations of GSH, which is present in tumor cells and in human tissues might, by binding and neutralizing the therapeutic compounds, reduce their effectiveness hence the need to modify these compounds to reduce their binding to $\mathrm{GSH}^{13}$. To accomplish this goal, we further modified CDODO-Me at $\mathrm{C}$ ring. By converting the carbonyl group in $\mathrm{C}$ ring into a dioen structure and generated methyl-2-cyano-3-oxo-18 $\beta$-olean1,9(11), 12-trien-30-oate (COOTO-Me, 10e, Fig. 1A) ${ }^{2}$. This compound only has minor decreased ability of inducing apoptosis comparing to the compound 10d but its ability to deplete the intracellular GSH is significantly decreased $^{2}$, providing an opportunity to identify the targets and the mechanisms of apoptosis induction without the involvement of GSH. 


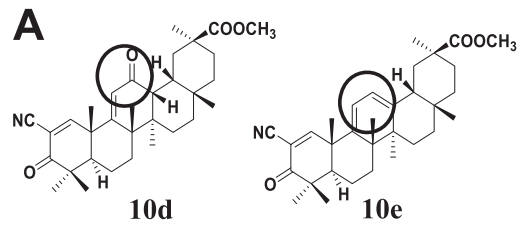

B

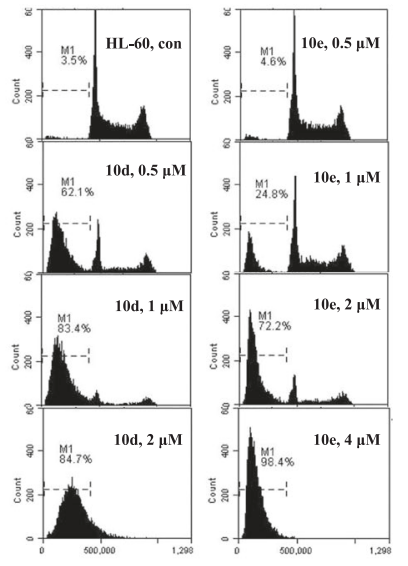

C
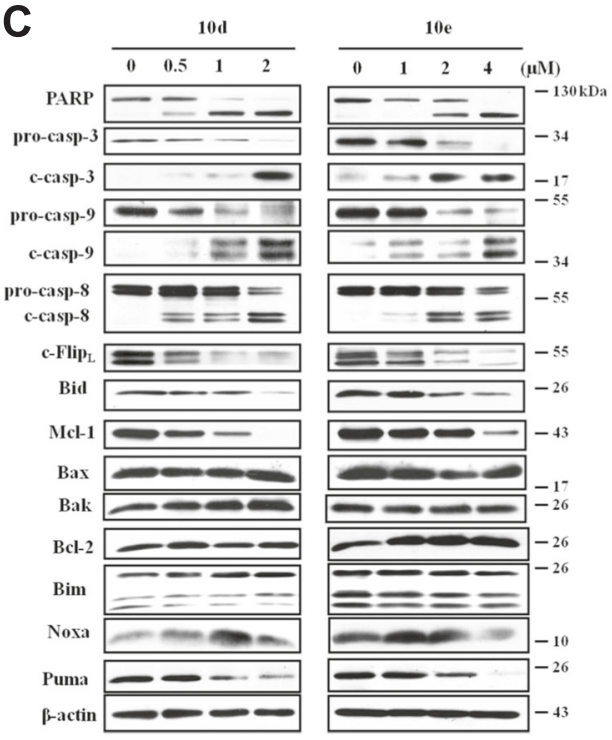

$\mathbf{F}$
D

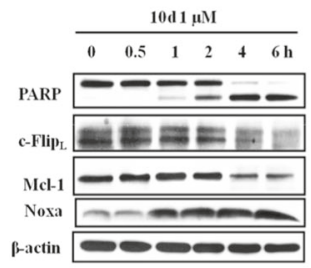

E

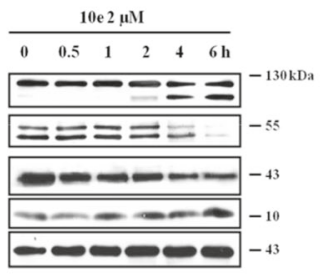

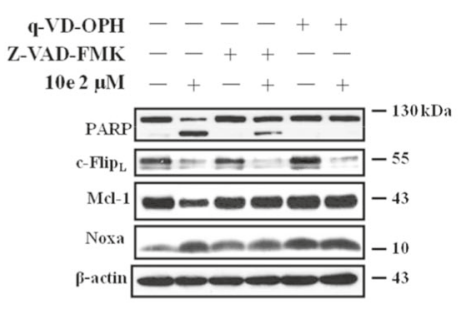

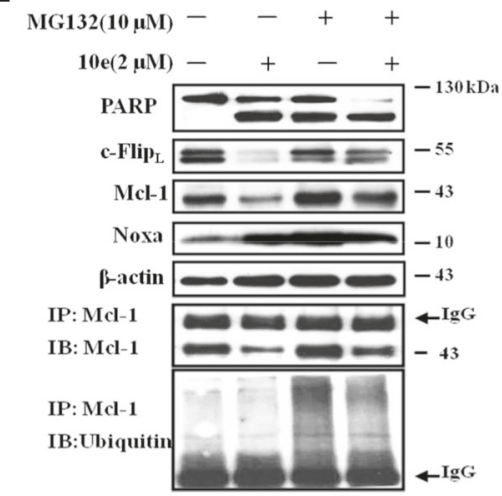

Fig. $1 \mathrm{10d}$ and 10e induce apoptosis in HL-60 cells associated with upregulation of Noxa and downregulation of Mcl-1 and c-Flip. A The chemical structures of $10 \mathrm{~d}$ and $10 \mathrm{e}$. $\mathbf{B} \mathrm{HL}-60$ cells were treated with $10 \mathrm{~d}$ and $10 \mathrm{e}$ at the indicated concentrations for $6 \mathrm{~h}$ and the cells in subG1 phase were determined using FACS analysis after staining with PI. con, untreated cells. C HL-60 cells treated with 10d or 10e at different concentrations for $6 \mathrm{~h}$, the relative levels of each protein were analyzed with western blotting using specific antibodies. D The HL-60 cells were treated with $1 \mu \mathrm{M} 10 \mathrm{~d}$ or $2 \mu \mathrm{M} 10 \mathrm{e}$ for the indicated time, the levels of PARP, C-Flip, Noxa proteins were examined. E HL-60 cells were pretreated with $100 \mu \mathrm{M}$ Z-VAD-FMK or $25 \mu \mathrm{M}$ q-VD-OPH for $1 \mathrm{~h}$, following by treatment with $2 \mu \mathrm{M} \mathrm{10e}$ for $6 \mathrm{~h}$, the relative levels of each protein were analyzed with western blotting using specific antibodies. F HL-60 cells were pretreated with $10 \mu \mathrm{M}$ MG132 for $1 \mathrm{~h}$ and then treated with or without $2 \mu \mathrm{M} 10 \mathrm{e}$ for $4 \mathrm{~h}$. The relative levels of PARP, C-Flip, Mcl-1, and Noxa were determined using Western blot analysis. The cell lysates was also immunoprecipitated with an anti-Mcl-1 antibody, and then immunoblotted with an anti-Mcl-1 antibody and an anti-ubiquitin antibody

The published mechanisms of apoptosis induction by CDODO-Me and CDDO-Me include an increase in reactive oxygen species (ROS), and the downregulation of the anti-apoptotic proteins Mcl-1 and c-Flip ${ }^{4,9,11}$. We did not find a role for the increased ROS in apoptosis induction in cells treated with 10e; in spite of this, treatment with 10e decreased c-Flip and Mcl-1 levels. c-Flip is a key protein blocking caspase- 8 activation, which controls extrinsic apoptosis pathway ${ }^{14}$, while Mcl-1 is one of the anti-apoptotic proteins controlling mitochondrial apoptotic pathway ${ }^{15}$. The latter is controlled by multiple anti- and pro-apoptotic proteins, and $\mathrm{BH} 3$-only proteins, which are currently being explored as therapeutic targets $^{16}$. We propose that apoptosis induction by $10 \mathrm{e}$ and $10 \mathrm{~d}$, as well as CDDO-Me is mediated by a new mechanism that leads to destruction of several antiapoptotic proteins. We explored this possibility by testing the key factors regulating both intrinsic and extrinsic apoptotic pathways in several human myeloid leukemia cell lines treated with 10e. Consistent with the reported downregulation of Mcl-1 and c-Flip, we found that Noxa was induced and that both Bax and Bak were activated. 
We further examined the mechanisms that might be responsible for the observed changes and found that the acetylation of Ku70 plays an essential role. We also found that $10 \mathrm{e}$ induces $\mathrm{Ku} 70$ acetylation by down-regulating HDAC3 and HDAC6 proteins. Our work reveals a novel mechanism of action of those modified compounds and provides a basis for further target-driven modifications of GA as potential cancer therapeutics.

\section{Results}

Both compound 10d and 10e induce apoptosis in HL-60 cells with downregulation of c-Flip and Mcl-1 and upregulation of Noxa protein

HL-60 cells were treated with varying concentrations of $10 \mathrm{~d}$ and $10 \mathrm{e}$ for $6 \mathrm{~h}$, the percent levels of apoptotic cells was determined using fluorescence-activated cell sorting (FACS) by measuring the percentages of the SubG1 fraction after staining with propidium iodide (PI) (Fig. 1B). More than $70 \%$ of the cells in the SubG1 phase were detected after treatment with $1 \mu \mathrm{M} 10 \mathrm{~d}$. To reach the same levels of apoptosis induction, $2 \mu \mathrm{M}$ 10e was required (Fig. 1B). The apoptotic effect of $10 \mathrm{e}$ is only slightly less than that of $10 \mathrm{~d}$ in this cell line, but is in the similar concentration range $(1-2 \mu \mathrm{M})$. Therefore, decreased ability of compound $10 \mathrm{e}$ as compared to $10 \mathrm{~d}$ to deplete intracellular $\mathrm{GSH}^{2}$, does not significantly affect its apoptosis inducing effect.

Western blot analysis was used to measure the apoptosis-related proteins in HL-60 cells treated with 10d and 10e. The cleaved caspase- $3,-8$, and -9 were detected in HL-60 cells, indicating that both extrinsic and intrinsic apoptotic pathways were involved in apoptosis induction after treatment with both compounds (Fig. 1C). As we reported before ${ }^{4}$, the levels of c-Flip and Mcl-1 were decreased while Bcl-2 was not changed in both $10 \mathrm{~d}$ and 10e-treated cells (Fig. 1C). The levels of Bak and Bax remained unchanged. BH3-only proteins are a group of proteins that includes, Bim, Noxa, and Puma, known to counteract the anti-apoptotic function of Bcl-2 family. Their regulation has not been tested in CDODO-Me and CDDO-Me treated cells. We measured the levels of Bim, Noxa, and Puma and found that Puma decreased, Noxa increased, while the level of Bim remained unchanged (Fig. 1C). These data suggest that downregulation of cFlip and Mcl-1, as well as the induction of Noxa is a part of the apoptosis inducing response to treatment with $10 \mathrm{~d}$ and 10e.

To explore in more detail the effect of $10 \mathrm{~d}$ and $10 \mathrm{e}$ on the three proteins, the cells were treated with $10 \mathrm{~d}$ or $10 \mathrm{e}$ and samples were retrieved at different times and examined for c-Flip and Mcl-1 and Noxa. The timing of apoptosis induction, measured by PARP cleavage, was correlated with a significant decrease in c-Flip and an increase in Noxa proteins (Fig. 1D). We then tested whether the regulation of Mcl-1, c-Flip, and Noxa by $10 \mathrm{e}$ was secondary to caspase activation or proteasome degradation. HL-60 cells were pretreated with pancaspase inhibitor Z-VAD-FMK and q-VD-OPH or proteasome inhibitor MG132. Pretreatment with Z-VADFMK or q-VD-OPH followed by $2 \mu \mathrm{M}$ 10e for $6 \mathrm{~h}$ attenuated the reduction in Mcl-1, but not in c-Flip, and did not affect the increase in Noxa level (Fig. 1E). Pretreatment of cells with MG132 enhanced apoptosis of 10e, in spite of the fact that the effects of 10e on c-Flip and Mcl-1 were diminished (Fig. 1F) (MG132 alone induced Noxa and partly induced apoptosis). To prove that MG132 at the used concentration inhibited proteasome, ubiquitinated Mcl-1 was measured using lysates immunoprecipitated with an anti-Mcl-1 antibody and detected in MG132 and MG132 with 10e-treated groups (Fig. 1F). These results suggest that c-Flip is down-regulated through the proteasome-mediated pathway while Mcl-1 level is controlled by both caspase and proteasomemediated pathways. Importantly, inhibition of proteasome activity appears to induce Noxa.

\section{Compound 10e is a weaker inhibitor than $10 \mathrm{~d}$ of AKT and ERK signaling pathways, which are known to regulate Mcl-1 stability}

CDDO-Me has been reported to inhibit AKT and mTOR signaling and ERK signaling, both known to regulate Mcl-1 stability through phosphorylation ${ }^{17}$. Components of both signaling pathways were examined after treatment of HL-60 cells with $10 \mathrm{~d}$ and 10e. The levels of total AKT, p-mTOR, p70S6K, and p-p70S6K, were decreased after treatment with $10 \mathrm{~d}$ at $1 \mu \mathrm{M}$ and $10 \mathrm{e}$ at 2 $\mu \mathrm{M}$. Neither 10d nor 10e influenced the levels of ERK and p-ERK. AKT phosphorylates GSK-3 $\beta$ on the $\operatorname{Ser}^{9}$ residue that leads to GSK-3 $\beta$ inactivation. Mcl-1 is phosphorylated by GSK-3 $\beta$ at $\operatorname{Ser}^{159}$, resulting in its proteasomal degradation $^{18}$. $10 \mathrm{~d}$ or $10 \mathrm{e}$ treatment led to reduction in the levels of phosphorylated GSK-3 $\beta$ on the $\operatorname{Ser}^{9}$ without changing GSK-3 $\beta$ protein levels (Sup. Figure 1). These data suggest that compounds $10 \mathrm{~d}$ and $10 \mathrm{e}$ can activate GSK-3 $\beta$ causing Mcl-1 degradation. Compared to 10d, 10 e was less effective in reducing Mcl-1 levels. Since Noxa is a protein which selectively binds to and inactivates Mcl1 , apoptosis induction might not depend on the downregulation of Mcl-1 in presence of upregulated Noxa.

\section{Downregulation of c-Flip by $10 \mathrm{e}$ is associated with the inhibition of histone deacetylases and Ku70 acetylation}

Inhibition of HDAC has been reported to decrease the levels of c-Flip ${ }^{19-21}$. c-Flip forms a stable complex with $\mathrm{Ku} 70$ that is dissociated after $\mathrm{Ku} 70$ acetylation $^{22}$. We tested the inhibitory effects of $10 \mathrm{~d}$ and $10 \mathrm{e}$ on the enzymatic activity of purified HDAC and found that, compared to MS-275, a selective inhibitor of HDAC activity, 
A
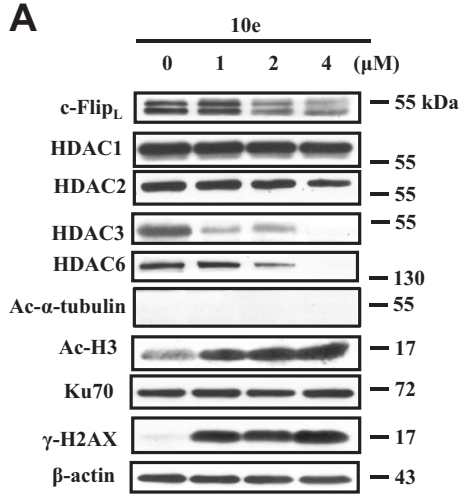

C

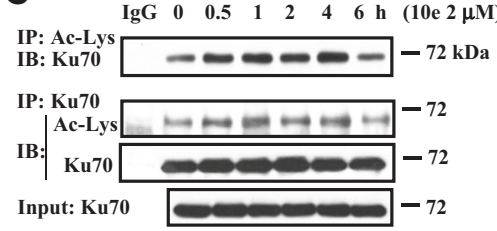

D

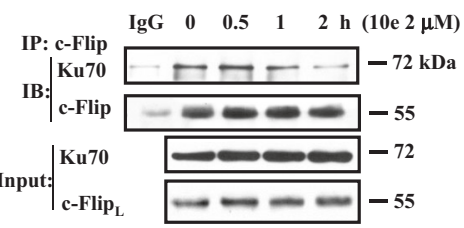

B
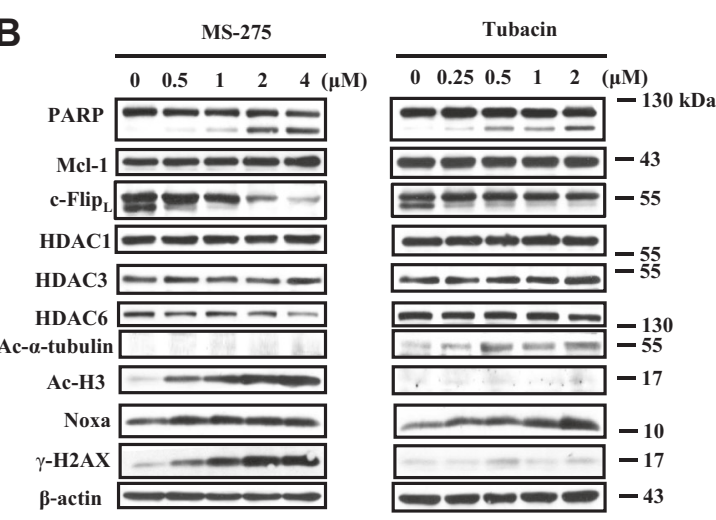

E

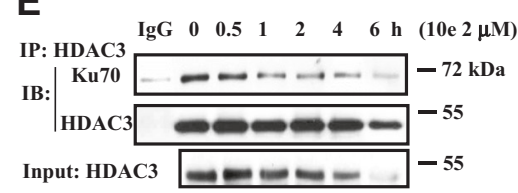

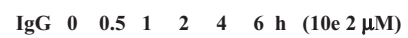

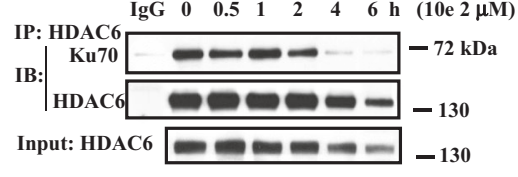

Fig. 2 10e decreased protein levels of c-Flip, HDAC3 and 6 and led to Ku70 acetylation. A HL-60 cells were treated with the indicated concentrations of 10 e for $6 \mathrm{~h}$, then the levels of the indicated proteins were determined using western blot analysis. B HL-60 cells were treated with MS-275 or tubacin at the indicated concentrations for $24 \mathrm{~h}$, then relative levels of the indicated proteins were determined by Western blotting. C, $\mathbf{D}$ The whole-cell lysates from HL-60 cells treated with $2 \mu \mathrm{M}$ 10e for the indicated time were immunoprecipitated with an acetylated lysine antibody, an Ku70 antibody (C) or with an c-Flip antibody (D), and then immunoblotted with an anti-Ku70, an acetylated lysine or an c-Flip antibody as labeled. E The cell lysates from HL-60 cells were immunoprecipitated with an anti-HDAC3 or anti-HDAC6 antibody and then immunoblotted for Ku70, HDAC3, HDAC6 proteins

neither compound was inhibitory (Sup. Figure 2). We then tested whether treatment of HL-60 cells with compound 10e has an effect on HDAC protein level. 10e decreased the levels of HDAC3 and HDAC6 proteins but not the levels of HDAC1 and HDAC2 (Fig. 2A). Only the acetylated $\mathrm{H} 3$ level, a substrate of HDAC3, was substantially increased; the substrate of HDAC6, $\alpha$-tubulin was not acetylated (Fig. 2A), suggesting that it is HDAC3 and not HDAC6 activity inhibition that contributes to the downregulation of c-Flip. To confirm this conclusion, we used the relatively specific HDAC inhibitor MS-275, which inhibits HDAC1, 2, and $3^{23}$, and tubacin, which is a relative selective inhibitor of $\mathrm{HDAC}^{24,25}$. As expected, MS-275 increased the levels of acetylated H3, but not acetylated $\alpha$-tubulin, while tubacin increased the levels of acetylated $\alpha$-tubulin, but not acetylated $\mathrm{H} 3$ at lower concentrations (Fig. 2B), indicating that it is possible to selectively inhibit either class I HDAC or HDAC6 at controlled concentrations. MS-275 was found to be more effective than tubacin in decreasing the levels of c-Flip and in apoptosis induction as measured by PARP cleavage. Interestingly we found that MS-275, like 10e, decreased the level of HDAC6 but without an increase in $\alpha$-tubulin acetylation. These data suggest that inhibition of HDAC1, 2, and 3 may lead to a decrease in the level of HDAC6. Inhibition of HDAC6 with tubacin has been reported to cause Ku70 acetylation and c-Flip degradation in colon cancer cells ${ }^{22}$. We increased the concentrations of tubacin and found that it indeed reduced the levels of cFlip protein in both HL-60 and THP-1 cells. However the reduced levels of c-Flip associated increased levels of acetylated H3 (Sup. Figure 3), suggesting that inhibition of HDAC1, 2, and 3, like MS-275, may contribute to tubacin-induced c-Flip reduction. The effect of compound 10e treatment on Ku70 acetylation was measured by immunoprecipitation (IP) using an acetylated lysine antibody and probed with an anti-Ku70 antibody and found to be increased as well as IP using an anti-Ku70 antibody and probed with the acetylated lysine antibody (Fig. 2C). This treatment also dissociated the c-Flip/Ku70 


\begin{tabular}{|c|c|c|c|c|c|c|c|c|}
\hline & $\begin{array}{c}\text { THP-1 } \\
10 \mathrm{e}\end{array}$ & & & $\begin{array}{c}\text { MOLM-13 } \\
10 \mathrm{e}\end{array}$ & \multicolumn{3}{|r|}{$\begin{array}{l}\text { NB4 } \\
10 \mathrm{e}\end{array}$} & \multirow[b]{2}{*}{$(\mu \mathrm{M})$} \\
\hline & $\begin{array}{lllll}0 & 2 & 4 & 6 & (1 \\
\end{array}$ & $\overline{(\mu} \mathbf{M})$ & & 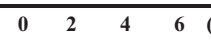 & $(\mu \mathrm{M})$ & & $\begin{array}{ll}0 & 2\end{array}$ & \\
\hline HDAC1 & 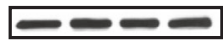 & $-55 \mathrm{kDa}$ & HDAC1 & $-\infty-\infty$ & $-55 \mathrm{kDa}$ & HDAC1 & $---\infty$ & $-55 \mathrm{kDa}$ \\
\hline HDAC3 & $=-\cdots$ & $日^{-55}$ & HDAC3 & $-1-$ & -55 & HDAC3 & $-2-1$ & -55 \\
\hline HDAC6 & --- & ]$_{-130}$ & HDAC6 & $-1-$ & -130 & HDAC6 & $E-$ & {$[-130$} \\
\hline PARP & $\Rightarrow \equiv=$ & ]$^{-130}$ & PARP & $-\equiv-$ & -130 & PARP & ED更 & -130 \\
\hline c-Flip ${ }_{L}$ & $\Leftrightarrow-\infty$ & -55 & c-Flip $\mathrm{L}_{\mathrm{L}}$ & $\equiv=\equiv$ & -55 & c-Flip & $=-=-$ & -55 \\
\hline Mcl-1 & 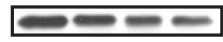 & -43 & Mcl-1 & 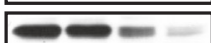 & $7-43$ & Mcl-1 & $-1-$ & -43 \\
\hline Noxa & $-\infty-\infty$ & -10 & Noxa & 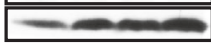 & -10 & Noxa & $=$ & -10 \\
\hline$\beta$-actin & $\infty$ & -43 & $\beta$-actin & $\Leftrightarrow$ & -43 & $\beta$-actin & $\Longrightarrow \infty$ & -43 \\
\hline & $\begin{array}{c}\text { U937 } \\
10 \mathrm{e}\end{array}$ & & & $\begin{array}{c}\text { KG-1 } \\
10 \mathrm{e}\end{array}$ & & & & \\
\hline & $\begin{array}{ll}0 & 2\end{array}$ & $(\mu \mathrm{M})$ & & $\begin{array}{ll}0 & 2\end{array}$ & $(\mu \mathrm{M})$ & & & \\
\hline HDAC1 & $\Leftrightarrow$ & $-55 \mathrm{kDa}$ & HDAC1 & $\Rightarrow$ & $-55 \mathrm{kDa}$ & & & \\
\hline HDAC3 & 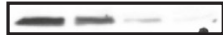 & ]$^{-55}$ & HDAC3 & $\sigma e=$ & -55 & & & \\
\hline HDAC6 & $--\cdots$ & ]-130 & HDAC6 & ---- & -130 & & & \\
\hline PARP & $-\approx \infty$ & -130 & PARP & $-\equiv \equiv \equiv$ & -130 & & & \\
\hline c-Flip & $-\infty-\cdots$ & ]-55 & c-Flip & $--\cdots-$ & -55 & & & \\
\hline Mcl-1 & -- & -43 & Mcl-1 & $=$ & -43 & & & \\
\hline Noxa & 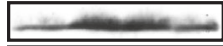 & ]$_{-10}$ & Noxa & 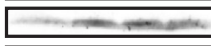 & -10 & & & \\
\hline$\beta$-actin & Cerar & -43 & $\beta$-actin & 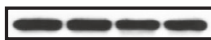 & -43 & & & \\
\hline $\begin{array}{l}\text { Fig. } 3 \text { The regulation } \\
\text { NB4, U937, and KG-1 cel } \\
\text { Western blotting }\end{array}$ & $\begin{array}{l}\text { f } 10 \mathrm{e} \text { on the prote } \\
\text { Is were treated with }\end{array}$ & $\begin{array}{l}\text { tein levels } \\
10 \mathrm{e} \text { at th }\end{array}$ & $\begin{array}{l}\text { f c-Flip, } \\
\text { indicate }\end{array}$ & $\begin{array}{l}\text { Mcl-1, Noxa, HD } \\
\text { d concentrations fo }\end{array}$ & $\begin{array}{l}\text { AC3, anc } \\
\text { or } 6 \mathrm{~h} \text {. Th }\end{array}$ & $\begin{array}{l}\text { HDAC6 i } \\
\text { levels of }\end{array}$ & $\begin{array}{l}\text { in different AML Ce } \\
\text { each indicated prot }\end{array}$ & $\begin{array}{l}\text { ell lines. THP-1, MOLM-13, } \\
\text { eein were determined with }\end{array}$ \\
\hline
\end{tabular}

complex before reduction in the c-Flip protein level (Fig. 2D). We measured the binding interaction of HDAC3 and HDAC6 with Ku70 in cell lysates treated with 10e. The binding of HDAC3 to Ku70 was decreased within $1 \mathrm{~h}$ of treatment with $10 \mathrm{e}$ while it took $2 \mathrm{~h}$ to dissociate the HDAC6/Ku70 complex (Fig. 2E). It has been described that inhibition of HDAC can cause DNA damage and an increased $\gamma-\mathrm{H} 2 \mathrm{AX}^{26}$. We measured the levels of $\gamma-\mathrm{H} 2 \mathrm{AX}$ in HL-60 cells treated with 10e, and found that, like in the treatment with MS-275, the $\gamma$ H2AX was increased by 10 e but not by tubacin at lower concentrations (Fig. 2A, B). The DNA damage caused by inhibition of HDAC1, 2, and 3, but not HDAC6 may be caused by induced acetylation of Ku70 in nucleus which might also contribute to apoptosis.

The findings that 10e treatment of HL-60 cells decreases the levels of c-Flip, Mcl-1, HDAC3, and HDAC6, while increasing the level of Noxa was found to be true in additional AML cell lines, such as THP-1, MOLM-13, NB4, U937, and KG-1 (Fig. 3).

\section{Noxa plays a crucial role in 10e-induced apoptosis in addition to Mcl-1 and c-Flip downregulation}

It has been described that induction of Noxa and downregulation of Mcl-1 causes mitochondria-mediated caspase9 activation $^{27}$. c-Flip is known to block apoptosis through interaction with caspase- $8^{28}$. To compare the roles of caspase- 8 and caspase- 9 in the apoptosis induced by $10 \mathrm{e}$, Jurkat subclones, A3 (expressing caspase-8) and I9.2 (deleted caspase-8) were used ${ }^{29}$. These cells were treated with $10 \mathrm{e}$ at the concentration of $4 \mu \mathrm{M}$; in subclone $\mathrm{A} 3$ (expressing caspase-8) this treatment caused $84.7 \%$ of cells to undergo apoptosis while in the 19.2 cells (deleted caspase-8) 53.7\% underwent apoptosis (Fig. 4A) (Apoptosis was measured by FACS analysis based on the Annexin V staining.). Western blot data showed that treatment with 10e decreased the c-Flip protein in both cell lines, while caspase- 8 was cleaved in A3 cells. These data suggest that caspase- 8 has a certain role in 10e-induced apoptosis since I9.2 cells, with deletion of caspase-8 decreased response to apoptosis induction comparing to A3 cells. The Mcl-1 level was decreased in A3 cells, but not in I9.2 cells after treatment with 10e. The levels of Noxa were similarly induced in both cell lines after 10e treatment. Silencing of Noxa significantly attenuated the apoptosis induced by $10 \mathrm{e}$ treatment in 19.2 cells (Fig. 4C), from 65 to 25\% (Fig. 4D). Similarly we found that silencing of Noxa attenuated the apoptosis induction by 10e in THP-1 cells (Sup. Figure 4A). These data suggest that Noxa induction but not Mcl-1 downregulation plays another crucial role for apoptosis induction by 10e treatment. Several factors such as FoxO3a and $\mathrm{CHOP}$ have been found to regulate the levels of Noxa expression $^{30}$. We found that the levels of FoxO3a were decreased while the levels of CHOP were induced in both 


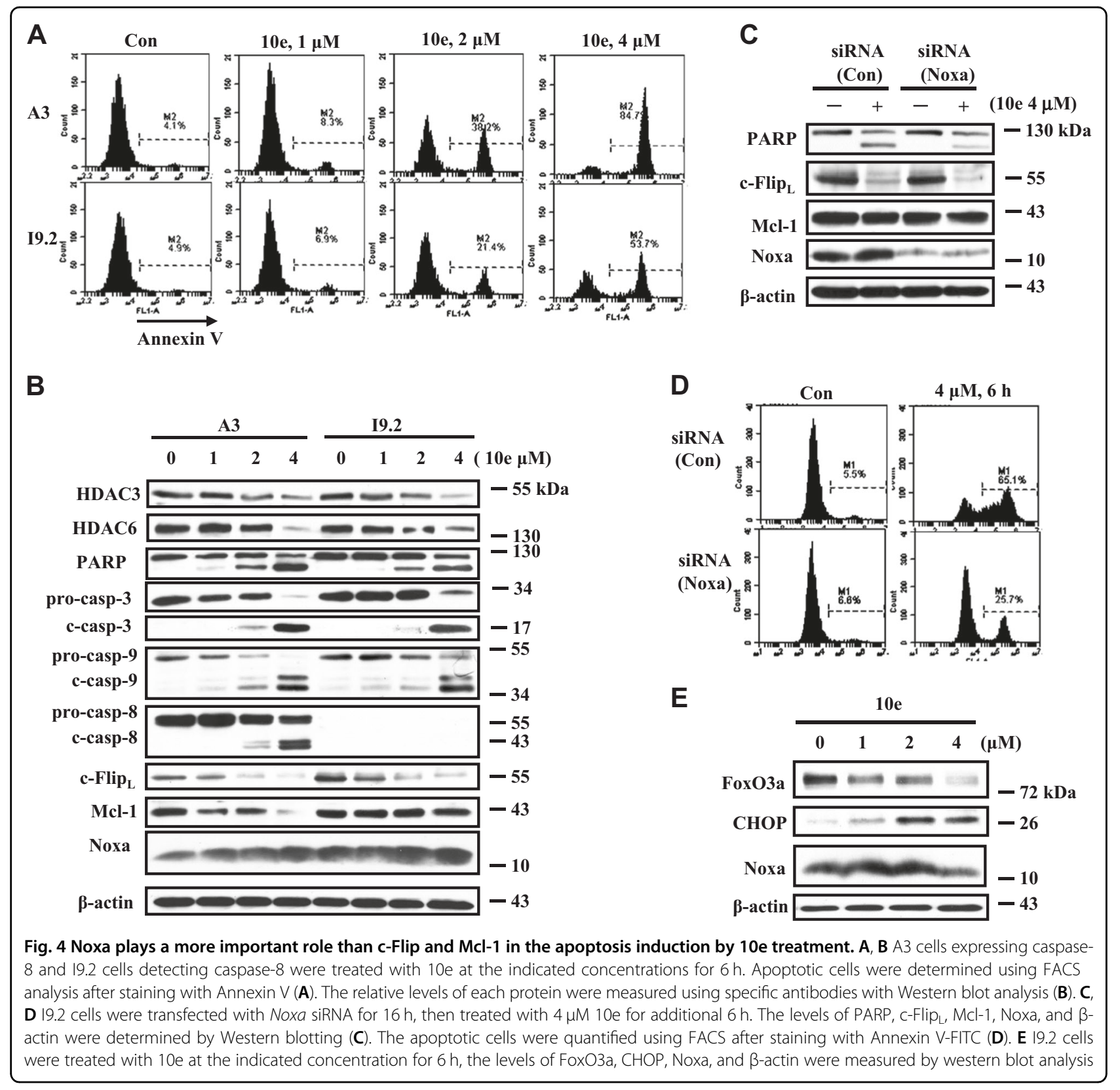

I9.2 cells (Fig. 4E) and THP-1 cells (Sup. Figure 4B), suggesting that a CHOP-mediated pathway leads to Noxa induction.

\section{Both Bak and Bax are activated in 10e-treated cells}

Noxa inactivates $\mathrm{Mcl}-1$ and leads to the activation of Bak. Since silencing of Noxa only partly blocked apoptosis in 19.2 cells treated with 10e (Fig. 4C) and in THP-1 cells (Sup. Figure 4A), it suggested that a non-Noxa/Mcl-1/Bak mediated pathway must also be involved in 10e-induced apoptosis. Using an IP assay, we found that both Bak and Bax were activated in THP-1, HL-60 cells, as well as in I9.2 cells (Fig. 5A). Silencing of Bak, similarly to silencing
Noxa (Sup. Figure 4A) attenuated apoptosis induced by 10e in THP-1 cells (Fig. 5B). Bax exists in free and bound forms with Bcl-2 or other proteins ${ }^{31}$. Bax binds to $\mathrm{Ku} 70$ and Ku70 acetylation can lead to Bax activation ${ }^{32,33}$. The interaction of Bax with $\mathrm{Ku} 70$ or $\mathrm{Bcl}-2$ was assessed by immunoprecipitating Bax and then probing with an antiKu70 or anti-Bcl-2 antibody. Interestingly, 10e treatment significantly increased the binding of Bax to Ku70 and Bcl-2 (Fig. 5C). Using the antibody detecting active form Bax, Bax (6A7), these interactions are not found, suggesting the bond form of Bax to $\mathrm{Ku} 70$ is not the active form. Silencing of Bax using siRNA decreased Bax protein, but neither the active Bax level nor apoptotic cells 


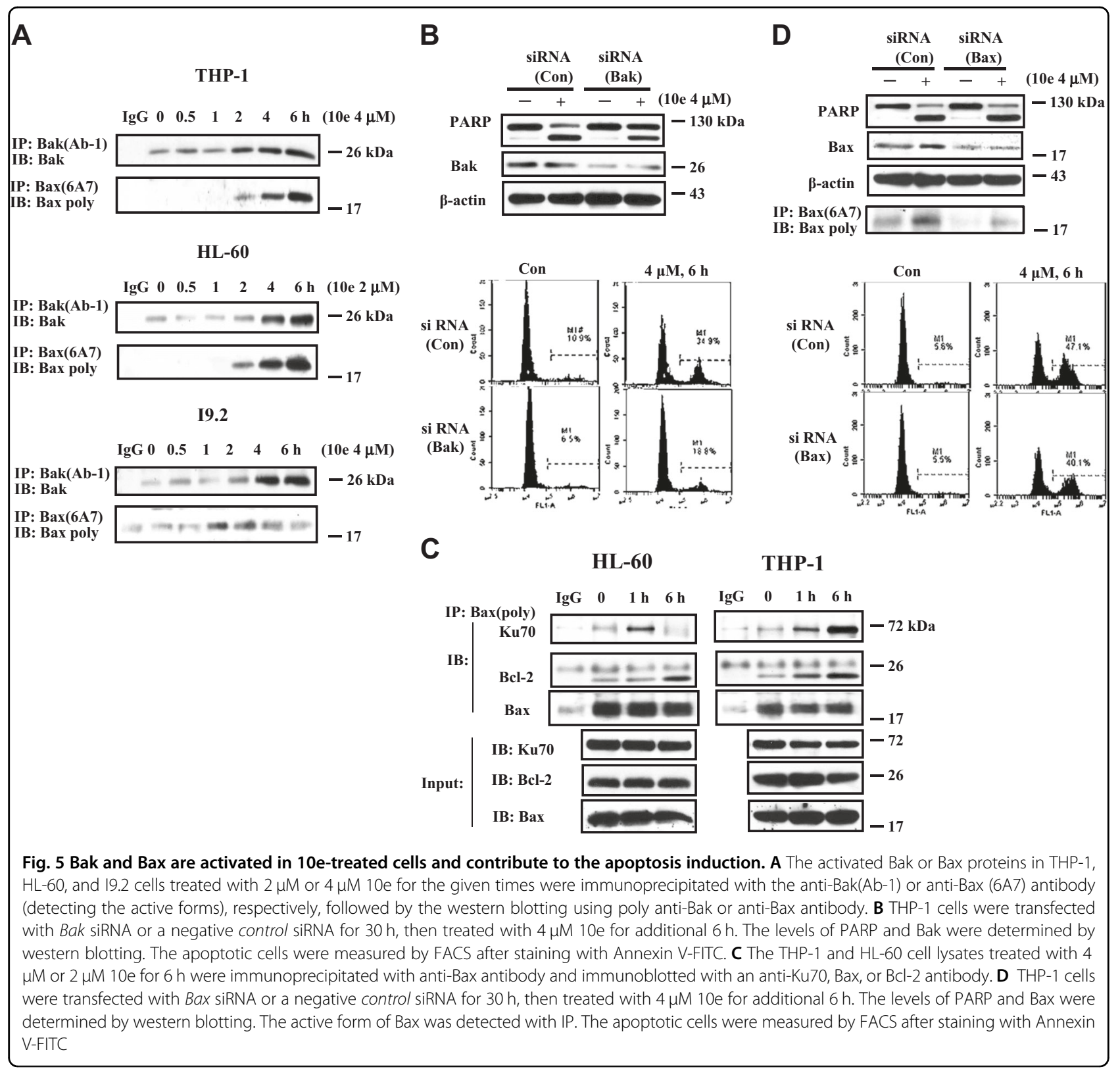

decreased in 10e-treated THP-1 cells (Fig. 5D). It has been reported that Ku70 by interacting with Bax stabilizes it by preventing its degradation ${ }^{33}$. We propose that $10 \mathrm{e}$ treatment regulates Bax through two ways: (1) increases its binding to non-acetylated Ku70 and (2) leads to Bax activation after dissociation from acetylated Ku70.

\section{Discussion}

Structural modified tritepenoids CDDO-Me and CDODO-Me are potent apoptosis inducers and are being developed for clinical trials of cancer therapy ${ }^{6,7}$. However, their development was delayed due to lack of clearly defined targets. Although, GSH depletion and ROS production have been thought to be the potential mechanisms of apoptosis induction ${ }^{9-12}$, the higher plasma GSH concentration and the role of GSH in redox regulation question GSH as a target ${ }^{34}$. Moreover the high affinity of these compounds to GSH put them into competitive disadvantage. We have found that $10 \mathrm{e}$ maintains apoptosis inducing ability of $10 \mathrm{~d}$ but with decreased binding to $\mathrm{GSH}^{2}$. We found that the following mechanisms contribute to the apoptosis induction by 10e: (1) Mcl-1 reduction and Noxa induction; (2) downregulation of c-Flip; and (3) Bax and Bak activation. By further exploring the mechanism causing those changes, we found new targets, HDAC3 and HDAC6. We showed that reduction of HDAC3 and HDAC6 proteins by 10e contribute to the apoptosis induction. 
c-Flip plays an important role in regulating extrinsic apoptosis by blocking the activation of caspase- 8 , while Noxa specifically binds to Mcl-1 and causes apoptosis through mitochondrial-mediated pathway ${ }^{27,35}$. Utilizing Jurkat subclones A3 cells (expressing caspase-8) and I9.2 cells (deleted caspase-8), we showed that 10e decreased the levels of c-Flip in both cell lines, and that I9.2 cells have less response to 10e-induced apoptosis than A3 cells (Fig. 4A), suggesting that c-Flip degradation caused caspase-8 activation play a role in the apoptosis induction. Compared to A3 cells, I9.2 cells contain high level of Mcl1 , which is not decreased by 10e treatment (Fig. 4B). Noxa was induced equally in both A3 and 19.2 cells after 10e treatment (Fig. 4B), but silencing of Noxa significantly decreased apoptosis in 10e-treated I9.2 cells (Fig. 4C) and THP-1 cells (Sup. Figure 4A). These data suggest that Noxa plays a more important role than Mcl-1 and that downregulation of Mcl-1 may not be required once Noxa is induced. This is supported by the observation that MG132 blocks Mcl-1 degradation induced by 10e treatment but enhances apoptosis by inducing Noxa (Fig. 1F).

We found that 10e-induced c-Flip degradation through a new HDAC/Ku70-mediated pathway. c-Flip interacts with $\mathrm{Ku} 70$ and is destabilized after Ku70 acetylation, which can be achieved by inhibition of $\mathrm{HDAC}^{22}$. We found that both HDAC3 and HDAC6 bind to Ku70 and dissociate from Ku70 once Ku70 is acetylated following 10e treatment (Fig. 2E). Although, 10e did not inhibit the activity of the pure HDAC enzyme (Sup. Fig. 2), it decreased the protein levels of HDAC3 and HDAC6 (Fig. 2A). Silencing of either HDAC3 or HDAC6 with siRNA augmented 10e-induced apoptosis in THP-1 cells (Sup. Figure 5). Interestingly, acetylated H3, a marker of inhibition of nuclear HDAC including HDAC3, but not the acetylated $\alpha$-tubulin, a marker of HDAC6 inhibition, was increased (Fig. 2A). These data suggest that inhibiting HDAC3, but not HDAC6, is important in causing Ku70 acetylation and c-Flip degradation. Indeed, we found that the relatively specific inhibitor of HDAC1, 2 and 3, MS275, decreased the levels of c-Flip, while the HDAC6 inhibitor, tubacin did not cause c-Flip degradation at the concentration only inhibiting HDAC6 based on the acetylated $\alpha$-tubulin (Fig. 2B). Interestingly MS-275 like 10e treatment decreased the levels of HDAC6 without increasing acetylated $\alpha$-tubulin (Fig. 2B), suggesting that HDAC3 might regulate the stability of HDAC6, a finding in need of further exploration. Ku70 not only binds to cFlip but also to Bax. Binding of Ku70 to Bax blocks its activation and translocation to mitochondria ${ }^{32,36,37}$. Ku70 acetylation disassociates Bax and leads to Bax activation $^{38,39}$. We found that treatment with 10e not only increased the levels of activated Bax (Fig. 5A) but also the binding of non-active Bax to Ku70 (Fig. 5C). Since 10e treatment increased the binding of Bax to both Ku70 and
Bcl-2 (Fig. 5C), we propose that this is a mechanism protecting a cell from death. It has been found that Ku70 can stabilize Bax through de-ubiquitination ${ }^{33}$. Therefore, 10e treatment simultaneously causes recruitment of Bax to Ku70 and also Bax activation in a portion after Ku70 acetylation. Several HDAC inhibitors have been approved for the treatment of cutaneous $\mathrm{T}$-cell lymphoma ${ }^{40}$. Although, these agents were originally developed as epigenetic regulators, they are potent apoptosis inducers through nonhistone targets. Recently it has been shown that bortezomib, an agent approved for treatment of myeloma, induces apoptosis while decreasing HDAC1, 2 and 3 protein levels ${ }^{41}$. It also has been shown that HDAC3 plays a more important role in cancer cells probably due to localization to the cytoplasm and the nucleus ${ }^{42}$ and its increased levels in some cancer cells ${ }^{43,44}$. Specific inhibitors of HDAC3 are being developed. We found that 10e treatment caused acetylation of Ku70 both in the nucleus and in the cytoplasm (Sup. Figure 6). Acetylation of Ku70 in cytoplasm is the cause of c-Flip degradation and Bax activation while its acetylation in the nucleus is the cause of DNA damage with increased levels of $\gamma$-H2AX (Fig. 2A, B).

It has been shown that the stability of $\mathrm{Mcl}-1$ is regulated by AKT/GSK-3 $\beta$ signaling and that Noxa is induced by the stress response protein $\mathrm{CHOP}^{45,46}$. We found that treatment with 10e induces CHOP (Fig. 4E and sup Fig. 4B) and decreases the AKT level. It has been previously shown that CDDO-Me is a HSP90 inhibitor and inhibition of HSP90 by several agents causes Noxa induction $^{47,48}$. Moreover, AKT is a client protein of HSP90 and the ability of HSP90 binding to client proteins is regulated by HDAC6. Therefore, the downregulation of Mcl-1 and AKT, as well as induction of Noxa may be due to inhibition of HSP90 as a response to decreased HDAC6 levels (Fig. 6). These connections need further study.

In summary, 10e is a novel GA derivative with apoptosis inducing ability and decreased binding to GSH. 10e induces apoptosis through multiple pathways: induction of Noxa and downregulation of c-Flip/Mcl-1, as well as activation of Bax and Bak (Fig. 6). We linked these events to the HDAC/Ku70 axis (Fig. 6). 10e functions as an HDAC3/6 inhibitor by reducing their protein levels rather than inhibiting their enzymatic activity. Our work reveals a novel mechanisms of CDDO-Me or CDODO-Me induced apoptosis which is important for directing further modification and optimization of those compounds as anticancer drugs.

\section{Materials and methods \\ Reagents}

$10 \mathrm{~d}$ and 10e were synthesized as we reported before; they were dissolved in dimethylsulfoxide for biological testing $^{2}$. The purity (more than 98\%) was determined using HPLC with a Hitachi UV detector L-2400 (Tokyo, 


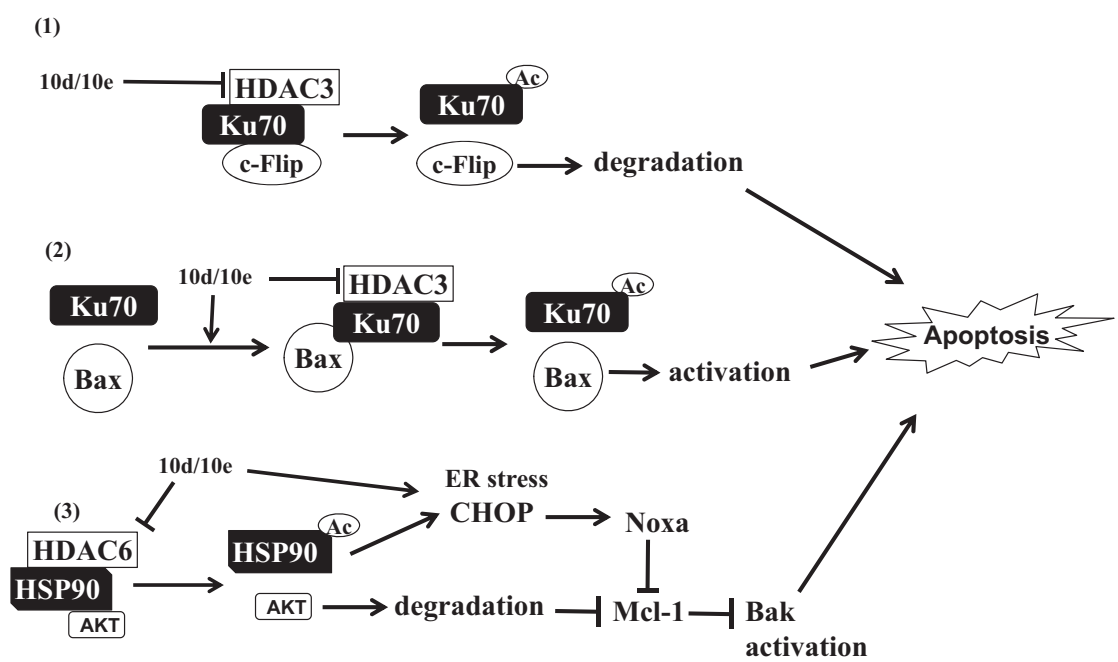

Fig. 6 Schematic presentation of apoptosis induction by 10e and 10d. 1 HDAC3/Ku70/c-Flip form a complex which is interrupted by 10e/10d through down-regulating HDAC3, resulting in c-Flip degradation; 2 10e/10d treatment increases the Ku70/Bax complex formation and, meanwhile, activates Bax by causing Ku70 acetylation through inhibiting HDAC3. 3 10e/10d treatment causes Noxa induction and AKT degradation by inhibiting or acetylating HSP90 through down-regulating HDAC6. Degraded AKT decreases Mcl-1 protein levels and Noxa induction inactivates Mcl-1, leading to Bak activation. The combined effects of c-Flip downregulation, Bax and Bak activation contribute to the apoptosis induction by $10 \mathrm{e} / 10 \mathrm{~d}$ treatment

Japan). MG132 were purchased from Sigma Chemical Co. (St. Louis, MO, USA). The pan-caspase inhibitor Z-VADFMK, MS-275, and tubacin were purchased from Selleckchem (Houston, TX). Antibodies to poly-(ADPribose)-polymerase(PARP), pro-caspase-3, and caspase-8 were obtained from BD Biosciences; to $\beta$-actin, Bid, Mcl1, Bcl-2, Bax (6A7), Ku70 (A-9), c-Flip, and Bax were purchased from Santa Cruz Biotechnology, Inc. (Santa Cruz, CA, USA); to procaspas-9, cleaved caspase-9, Bim, Puma, CHOP, FoxO3a, ERK, p-ERK, eIF4E, p-eIF4E, AKT, p-AKT, mTOR, p-mTOR, 4E-BP1, p-4E-BP1, p70S6K, p-p70S6K $\left(\right.$ Thr $\left.^{389}\right)$, GSK-3 $\beta, \quad$-GSK-3 $\beta\left(\right.$ Ser $\left.^{9}\right)$, Acetyl- $\alpha-$ Tubulin, Bax, Bak, acetylated lysine, and $\gamma-$ H2AX were purchased from Cell Signaling Technology, Inc. (Berverly, MA, USA); to Noxa and Ku70 were obtained from Abcam, Inc. (Cambridge, MA, USA); to acetyl-Histone $\mathrm{H} 3$ was from Active Motif. (Carlsbad, CA, USA), to Bak (Ab-1) was obtained from Merck Millipore (Merck, Darmstadt, Germany). Noxa, Bak, Bax, HDAC3, HDAC6 siRNA, and a control siRNA were purchased from Santa Cruz Biotechnology, Inc.

\section{Cell culture}

Acute myeloid leukemia HL-60, MOLM-13, THP-1, U937, KG-1 cells, and acute promyelocytic leukemia NB4 cells were cultured in RPMI 1640 medium supplemented with $100 \mathrm{units} / \mathrm{mL}$ penicillin, $100 \mu \mathrm{g} / \mathrm{mL}$ streptomycin, 1 $\mathrm{mmol} / \mathrm{L} \mathrm{L}$-glutamine, and $10 \%(\mathrm{v} / \mathrm{v})$ heat-inactivated fetal bovine serum (FBS). A3 and its caspase- 8 deficient I9.2 cells were subclones of human Jurkat cells which were cultured in RPMI 1640 modified to contain $2 \mathrm{mmol} / \mathrm{L} \mathrm{L-}$ glutamine, $10 \mathrm{mmol} / \mathrm{L}$ HEPES, $1.0 \mathrm{mmol} / \mathrm{L}$ sodium pyruvate, $4.5 \mathrm{~g} / \mathrm{L}$ glucose, and $1.5 \mathrm{~g} / \mathrm{L}$ sodium bicarbonate, 100 units $/ \mathrm{mL}$ penicillin, $100 \mu \mathrm{g} / \mathrm{mL}$ streptomycin, and $10 \%(\mathrm{v} / \mathrm{v})$ heat-inactivated FBS.

\section{Quantitation of apoptotic cells}

Apoptotic cells were quantified by FACS analysis after staining with PI and annexin V. For FACS analysis with PI staining, cells were fixed with $70 \%$ ice-cold ethanol at a density of $1 \times 10^{5}$ cells $/ \mathrm{mL}$ and treated by the PI assay kit according to the manufacturer's instructions (BD Biosciences). For FACS analysis with annexin V staining, Annexin V-FITC Apoptosis Detection Kit (BD Biosciences) was used.

\section{IP}

Cells were collected, washed with PBS and lysed using ice-cold NP-40 lysis buffer ( $50 \mathrm{mmol} / \mathrm{L}$ Tris- $\mathrm{HCl}$ (pH 7.5), $150 \mathrm{mmol} / \mathrm{L} \mathrm{NaCl}, 5 \mathrm{mmol} / \mathrm{L}$ EDTA, $0.5 \% \mathrm{NP}-40,50$ $\mathrm{mmol} / \mathrm{L} \mathrm{NaF}, 0.2 \mathrm{mmol} / \mathrm{L} \mathrm{Na} \mathrm{VO}_{4}, 1 \mathrm{mmol} / \mathrm{L} \mathrm{DTT}$ ) for $60 \mathrm{~min}$ on ice. Total protein $(400 \mu \mathrm{g})$ was first precleared with $20 \mu \mathrm{l}$ protein A/G plus-agarose (Santa Cruz Biotechnology) and then subjected to IP with 1-2 $\mu$ g of either normal IgG or specific antibody at $4{ }^{\circ} \mathrm{C}$ for $2 \mathrm{~h}$. Then $20 \mu \mathrm{l}$ of protein A/G plus-agarose beads were added and incubated overnight to pull down protein-antibody complexes. The beads were spun, washed four times with NP40 lysis buffer, resuspended in 2X SDS sample buffer (50 $\mathrm{mM}$ Tris- $\mathrm{HCl}(\mathrm{pH}$ 6.8), $2 \%$ SDS, $10 \%$ glycerol, $5 \% \beta-$ mercaptoethanol) and heated at $98{ }^{\circ} \mathrm{C}$ for $5 \mathrm{~min}$ for analysis by SDS-polyacrylamide gel electrophoresis and Western blotting. 


\section{Nuclear and cytoplasmic extraction assay}

Cytoplasmic and nuclear lysates of HL-60 cells were prepared using NE-PER Nuclear and cytoplasmic extraction reagent (\#78833, Thermo Scientific, Rockford, IL) and subjected to western blot analysis and IP.

Western blot analysis, detection of activated Bak and Bax based conformational changes, as well as siRNA use to silence gene expression were done as we reported before $^{49}$. The HDAC activity was measured as reported before $^{50}$.

\section{Acknowledgements}

This work was partly supported by The Startup Fund of Shenyang Pharmaceutical University, The Samuel Waxman Cancer Research Foundation. We thank Dr. Liliana Ossowski for reading of this manuscript.

\section{Author details}

'Department of Pharmacology, Shenyang Pharmaceutical University, Shenyang 110016, PR China. ${ }^{2}$ Department of Medicinal Chemistry, Shenyang Pharmaceutical University, Shenyang 110016, PR China. ${ }^{3}$ Key Laboratory of Structure-Based Drug Design \& Discovery of Ministry of Education, Shenyang Pharmaceutical University, Shenyang 110016, PR China

\section{Conflict of interest}

The authors declare that they have no conflict of interest.

\section{Publisher's note}

Springer Nature remains neutral with regard to jurisdictional claims in published maps and institutional affiliations.

Supplementary Information accompanies this paper at (https://doi.org/ 10.1038/s41419-018-0602-1).

Received: 19 December 2017 Revised: 25 March 2018 Accepted: 16 April 2018

Published online: 24 May 2018

\section{References}

1. Wang, Z. Y. \& Nixon, D. W. Licorice and cancer. Nutr. Cancer 39, 1-11 (2001).

2. Li, X. et al. Synthesis of methyl 2-cyano-3,12-dioxo-18beta-olean-1,9(11)-dien30 -oate analogues to determine the active groups for inhibiting cell growth and inducing apoptosis in leukemia cells. Org. Biomol. Chem. 12, 6706-6716 (2014).

3. Logashenko, E. B. et al. Synthesis and pro-apoptotic activity of novel glycyrrhetinic acid derivatives. Chembiochem 12, 784-794 (2011).

4. Song, D. et al. Downregulation of c-FLIP, XIAP and Mcl-1 protein as well as depletion of reduced glutathione contribute to the apoptosis induction of glycyrrhetinic acid derivatives in leukemia cells. Cancer Biol. Ther. 9, 96-108 (2010).

5. Chadalapaka, G., Jutooru, I., McAlees, A., Stefanac, T. \& Safe, S. Structuredependent inhibition of bladder and pancreatic cancer cell growth by 2substituted glycyrrhetinic and ursolic acid derivatives. Bioorg. Med. Chem. Lett. 18, 2633-2639 (2008)

6. Shanmugam, M. K. et al. Oleanolic acid and its synthetic derivatives for the prevention and therapy of cancer: preclinical and clinical evidence. Cancer Lett. 346, 206-216 (2014).

7. Petronelli, A., Pannitteri, G. \& Testa, U. Triterpenoids as new promising anticancer drugs. Anticancer Drugs 20, 880-892 (2009).

8. Zou, W. et al. c-FLIP downregulation contributes to apoptosis induction by the novel synthetic triterpenoid methyl-2-cyano-3, 12-dioxooleana-1, 9-dien-28oate (CDDO-Me) in human lung cancer cells. Cancer Biol. Ther. 6, 1614-1620 (2007).

9. Yue, P., Zhou, Z., Khuri, F. R. \& Sun, S. Y. Depletion of intracellular glutathione contributes to JNK-mediated death receptor 5 upregulation and apoptosis induction by the novel synthetic triterpenoid methyl-2-cyano-3, 12-dioxooleana-1, 9-dien-28-oate (CDDO-Me). Cancer Biol. Ther. 5, 492-497 (2006).

10. Samudio, I. et al. 2-Cyano-3,12-dioxooleana-1,9-dien-28-imidazolide (CDDO-Im) directly targets mitochondrial glutathione to induce apoptosis in pancreatic cancer. J. Biol. Chem. 280, 36273-36282 (2005).

11. Ikeda, T., Sporn, M., Honda, T., Gribble, G. W. \& Kufe, D. The novel triterpenoid CDDO and its derivatives induce apoptosis by disruption of intracellular redox balance. Cancer Res. 63, 5551-5558 (2003).

12. Ikeda, T. et al. Induction of redox imbalance and apoptosis in multiple myeloma cells by the novel triterpenoid 2-cyano-3,12-dioxoolean-1,9-dien-28-oic acid. Mol. Cancer Ther. 3, 39-45 (2004).

13. Boysen, G. The glutathione conundrum: stoichiometric disconnect between its formation and oxidative stress. Chem. Res. Toxicol. 30, 1113-1116 (2017).

14. Fulda, S. Targeting C-FLICE-like inhibitory protein (CFLAR) in cancer. Expert. Opin. Ther. Targets 17, 195-201 (2013).

15. Fernandez-Marrero, Y., Spinner, S., Kaufmann, T. \& Jost, P. J. Sunvival control of malignant lymphocytes by anti-apoptotic MCL-1. Leukemia 30, 2152-2159 (2016).

16. Opferman, J. T. Attacking cancer's Achilles heel: antagonism of anti-apoptotic BCL-2 family members. FEBS J. 283, 2661-2675 (2016).

17. Deeb, D., Gao, X., Jiang, H., Dulchavsky, S. A. \& Gautam, S. C. Oleanane triterpenoid CDDO-Me inhibits growth and induces apoptosis in prostate cancer cells by independently targeting pro-survival Akt and mTOR. Prostate 69, 851-860 (2009).

18. Inuzuka, H. et al. Mcl-1 ubiquitination and destruction. Oncotarget 2, 239-244 (2011).

19. Carson, R. et al. HDAC inhibition overcomes acute resistance to MEK inhibition in BRAF-mutant colorectal cancer by downregulation of c-FLIPL. Clin. Cancer Res. 21, 3230-3240 (2015).

20. Venza, I., Visalli, M., Oteri, R., Teti, D. \& Venza, M. Class I-specific histone deacetylase inhibitor MS-275 overrides TRAll-resistance in melanoma cells by downregulating C-FLIP. Int. Immunopharmacol. 21, 439-446 (2014).

21. Hurwitz, J. L. et al. Vorinostat/SAHA-induced apoptosis in malignant mesothelioma is FLIP/caspase 8-dependent and HR23B-independent. Eur. J. Cancer 48, 1096-1107 (2012).

22. Kerr, E. et al. Identification of an acetylation-dependant Ku70/FLIP complex that regulates FLIP expression and HDAC inhibitor-induced apoptosis. Cell Death Differ. 19, 1317-1327 (2012).

23. Ruiz, R., Raez, L. E. \& Rolfo, C. Entinostat (SNDX-275) for the treatment of nonsmall cell lung cancer. Expert Opin. Investig. Drugs 24, 1101-1109 (2015).

24. Simms-Waldrip, T. et al. The aggresome pathway as a target for therapy in hematologic malignancies. Mol. Genet. Metab. 94, 283-286 (2008).

25. Butler, K. V. et al. Rational design and simple chemistry yield a superior, neuroprotective HDAC6 inhibitor, tubastatin A. J. Am. Chem. Soc. 132 10842-10846 (2010).

26. Wilson, A. J. et al. The DNA damage mark pH2AX differentiates the cytotoxic effects of small molecule HDAC inhibitors in ovarian cancer cells. Cancer Biol. Ther. 12, 484-493 (2011).

27. Guikema, J. E., Amiot, M. \& Eldering, E. Exploiting the pro-apoptotic function of NOXA as a therapeutic modality in cancer. Expert Opin. Ther. Targets 21 767-779 (2017).

28. Kataoka, T. The caspase-8 modulator c-FLIP. Crit. Rev. Immunol. 25, 31-58 (2005).

29. Lombard, C., Nagarkatti, M. \& Nagarkatti, P. S. Targeting cannabinoid receptors to treat leukemia: role of cross-talk between extrinsic and intrinsic pathways in Delta9-tetrahydrocannabinol (THC)-induced apoptosis of Jurkat cells. Leuk. Res. 29, 915-922 (2005).

30. Ploner, C., Kofler, R. \& Villunger, A. Noxa: at the tip of the balance between life and death. Oncogene 27(Suppl 1), S84-S92 (2008).

31. Walensky, L. D. \& Gavathiotis, E. BAX unleashed: the biochemical transformation of an inactive cytosolic monomer into a toxic mitochondrial pore. Trends Biochem. Sci. 36, 642-652 (2011).

32. Hada, M. \& Kwok, R. P. Regulation of ku70-bax complex in cells. J. Cell Death $\mathbf{7}$ 11-13 (2014).

33. Amsel, A. D., Rathaus, M., Kronman, N. \& Cohen, H. Y. Regulation of the proapoptotic factor Bax by Ku70-dependent deubiquitylation. Proc. Natl Acad. Sci. USA 105, 5117-5122 (2008).

34. Circu, M. L. \& AW, T. Y. Glutathione and modulation of cell apoptosis. Biochim. Biophys. Acta 1823, 1767-1777 (2012).

35. Safa, A. R. c-FLIP, a master anti-apoptotic regulator. Exp. Oncol. 34, 176-184 (2012). 
36. Gomez, J. A. et al. Bax-inhibiting peptides derived from Ku70 and cellpenetrating pentapeptides. Biochem. Soc. Trans. 35, 797-801 (2007).

37. Li, Y. et al. Bax-inhibiting peptide protects cells from polyglutamine toxicity caused by Ku70 acetylation. Cell Death Differ. 14, 2058-2067 (2007).

38. Subramanian, C., Opipari, A. W. Jr., Bian, X., Castle, V. P. \& Kwok, R. P. Ku70 acetylation mediates neuroblastoma cell death induced by histone deacetylase inhibitors. Proc. Natl Acad. Sci. USA 102, 4842-4847 (2005).

39. Subramanian, C., Jarzembowski, J. A., Opipari, A. W. Jr., Castle, V. P. \& Kwok, R. P. HDAC6 deacetylates Ku70 and regulates Ku70-Bax binding in neuroblastoma. Neoplasia 13, 726-734 (2011).

40. Ocker, M. Deacetylase inhibitors - focus on non-histone targets and effects. World J. Biol. Chem. 1, 55-61 (2010).

41. Kikuchi, J. et al. Histone deacetylases are critical targets of bortezomib-induced cytotoxicity in multiple myeloma. Blood 116, 406-417 (2010).

42. Mano, T., Suzuki, T., Tsuji, S. \& Iwata, A. Differential effect of HDAC3 on cytoplasmic and nuclear huntingtin aggregates. PLOS ONE 9, e111277 (2014).

43. Minami, J. et al. Histone deacetylase 3 as a novel therapeutic target in multiple myeloma. Leukemia 28, 680-689 (2014).
44. Stengel, K. R. et al. Histone deacetylase 3 is required for efficient T cell development. Mol. Cell. Biol. 35, 3854-3865 (2015).

45. Ruvolo, P. P. GSK-3 as a novel prognostic indicator in leukemia. Adv. Biol. Regul. 65, 26-35 (2017)

46. Iurlaro, R. \& Munoz-Pinedo, C. Cell death induced by endoplasmic reticulum stress. FEBS J. 283, 2640-2652 (2016).

47. Qin, D. J. et al. Hsp90 Is a novel target molecule of CDDO-Me in inhibiting proliferation of ovarian cancer cells. PLOS. ONE 10, e0132337 (2015).

48. Davis, A. L. et al. The quinone methide aurin is a heat shock response inducer that causes proteotoxic stress and Noxa-dependent apoptosis in malignant melanoma cells. J. Biol. Chem. 290, 1623-1638 (2015).

49. Wang, R., Xia, L., Gabrilove, J., Waxman, S. \& Jing, Y. Downregulation of Mcl-1 through GSK-3beta activation contributes to arsenic trioxide-induced apoptosis in acute myeloid leukemia cells. Leukemia 27, 315-324 (2013).

50. Baud, M. G. et al. Defining the mechanism of action and enzymatic selectivity of psammaplin A against its epigenetic targets. J. Med. Chem. 55, 1731-1750 (2012). 